\title{
Detection of zero anisotropy at 5.2 AU during the November 1998 solar particle event: Ulysses Anisotropy Telescopes observations
}

\author{
S. Dalla, A. Balogh \\ The Blackett Laboratory, Imperial College, London, SW7 2BZ, UK \\ E-mail: s.dalla@ic.ac.uk \\ Received: 19 July 1999 / Revised: 15 November 1999 / Accepted: 30 November 1999
}

\begin{abstract}
For the first time during the mission, the Anisotropy Telescopes instrument on board the Ulysses spacecraft measured constant zero anisotropy of protons in the 1.3-2.2 MeV energy range, for a period lasting more than three days. This measurement was made during the energetic particle event taking place at Ulysses between 25 November and 15 December 1998, an event characterised by constant high proton fluxes within a region delimited by two interplanetary forward shocks, at a distance of 5.2 AU from the Sun and heliographic latitude of $17^{\circ} \mathrm{S}$. We present the ATs results for this event and discuss their possible interpretation and their relevance to the issue of intercalibration of the two telescopes.
\end{abstract}

Key words: Interplanetary physics (energetic particles) Solar physics, astrophysics and astronomy (energetic particles) - Space plasma physics (instruments and techniques)

\section{Introduction}

The Ulysses spacecraft completed its first polar orbit around the Sun on April 17, 1998, at a distance of 5.4 $\mathrm{AU}$ from the Sun and heliographic latitude $6^{\circ} \mathrm{S}$. During the second half of 1998, in correspondence with the increase in solar activity towards solar maximum, several large energetic particle events were detected by the Anisotropy Telescopes (ATs) and other instruments of the Cosmic Ray and Solar Particle Investigation (COSPIN) (Simpson et al., 1992) on board the spacecraft. The ATs are a two-telescope sectored multichannel system detecting protons in the energy range 1.3-3.6 MeV and $\alpha$-particles in the range $3-26 \mathrm{MeV}$ (Staines et al., 1993). The sectored counts allow recon-

Correspondence to: S. Dalla struction of particle flow directions by means of a spherical harmonic analysis.

The acceleration and propagation of energetic particles in the heliosphere have been the subject of extensive experimental and theoretical efforts for a number of years. During the first phase of the Ulysses mission, work on $\mathrm{MeV}$ energy particles has focused mainly on recurrent 26-day particle events associated with corotating interaction regions, taking place during the declining phase of solar cycle 22 (e.g. Sanderson et al., 1994; Simnett et al., 1995). As solar activity started to increase towards the maximum of cycle 23 , transient CME-associated particle events have been detected at Ulysses (Marsden et al., 1999), starting with an isolated event in November 1997 (Lario et al., 1998).

We focus on a specific particle event taking place at Ulysses between 25 November and 14 December, 1998, during which the ATs recorded, for the first time during the mission, a period of zero particle flows (zero anisotropy) in the solar wind frame lasting for approximately 3.5 days. At this time the spacecraft was located at $5.2 \mathrm{AU}$ from the Sun and heliographic latitude $17^{\circ} \mathrm{S}$. The magnitude of the first order anisotropy during the whole period remained below values of 0.08 , and, unusually, showed very little variability. Anisotropies of protons in the interplanetary space have been the subject of extensive studies (e.g. Sanderson et al., 1985 and references therein). Typically, anisotropies in the spacecraft frame are the superposition of the Compton-Getting anisotropy, which results from the solar wind being convected past the spacecraft at the solar wind speed (Ipavich, 1974), and of anisotropies resulting from particle streaming. Here we are assuming that anisotropies resulting from spatial gradients can be neglected. Transformation of the anisotropies into the solar wind frame, by subtraction of the Compton-Getting anisotropy, allows one to focus on particle streaming. In the solar wind frame particle anisotropies are generally gyrotropic about the magnetic field direction and in most cases the component of the first order anisotropy perpendicular to the magnetic field direction is negligible for protons in the $\mathrm{MeV}$ range. 
As far as we are aware an event with constant zero anisotropy in the solar wind frame, such as the one we present here, has not previously been reported. We present anisotropy data for three sectored proton channels and discuss possible interpretations of the zero anisotropy phase.

In addition, we use our anisotropy measurement to calculate the speed of the rest frame, defined as the frame in which the particle distribution function is isotropic and has a power law energy spectrum (Tan et al., 1988). Assuming that wave scattering is responsible for particle isotropy, the rest frame is the frame comoving with the waves. We find that the rest frame is moving very slowly with respect to the solar wind during the times of lowest anisotropy, but can reach speeds of the order of $100 \mathrm{~km} / \mathrm{s}$ already at anisotropies of $\sim 0.08$ for the case of hard spectra.

Finally, we comment on the implications the zero anisotropy event bears on the issue of intercalibration of the two ATs telescopes.

\section{Measurement and data analysis}

We consider $\mathrm{MeV}$ ion data from the Anisotropy Telescopes (ATs) instrument, forming part of the COSPIN package on board the Ulysses spacecraft. The ATs instrument has been discussed in detail by Simpson et al. (1992), with important revisions regarding the calibration of the energy channels and orientation of the telescopes with respect to the spacecraft spin axis reported by Staines et al. (1993).

The ATs comprise two identical solid state particle detectors with geometrical factors of $\sim 0.7 \mathrm{~cm}^{2}$ sr and full cone opening angles of $70^{\circ}$ mounted at angles of $60^{\circ}$ (telescope 1; T1) and $145^{\circ}$ (telescope 2; T2) to the spacecraft spin axis. Ion fluxes incident on the telescopes are divided into a number of data channels, sensitive mainly to protons and alpha particles. Protons are detected in the energy range $0.7-6.7 \mathrm{MeV}$. Four of the thirteen channels available in each telescope separate the counts into eight azimuthal sectors as the spacecraft spins, thus allowing a reconstruction of the particle flows by means of the spherical harmonic analysis described later.

We focus on data from three sectored channels detecting protons in the ranges 1.3-2.2 MeV (channel 7), 2.2-3.6 MeV (channel 8) and 3.6-6.7 MeV (channel 9). These channels are in fact also sensitive to $\alpha$-particles, but analysis of proton and $\alpha$ spectra obtained by means of proton-only, $\alpha$-only and mixed species channels shows that the main contribution to the fluxes for the event under consideration is from protons.

The anisotropy is derived from the ATs measurement as follows. The two sectored channels (one for each telescope) of centre energy $E$ provide a total of sixteen directional differential flux measurements. We indicate as $J\left(E, \mathbf{e}_{v}\right)$ the measured differential flux (in $\left(\mathrm{cm}^{2} \mathrm{sr} \mathrm{s}\right.$ $\mathrm{MeV})^{-1}$ ) at energy $E$ along the direction identified by the unit vector $\mathbf{e}_{v}$, taken as pointing towards the centre of the sector under consideration. We assume that the differential flux can be fitted by means of a reduced second-order spherical harmonic expansion as follows:

$J\left(E, \mathbf{e}_{v}\right)=J_{0}(E)\left[1+\mathbf{A}_{l}(E) \cdot \mathbf{e}_{v}+A_{2}(E) \frac{3 \mu^{2}-1}{2}\right]$

where $\mu=\cos \Psi$ and $\Psi$ is the pitch-angle of detected ions, i.e. the angle between the magnetic field and the vector $\mathbf{e}_{v}$, and we have assumed the second order harmonics to be gyrosymmetric about the field direction. The magnetic field data used in this analysis is provided by the Ulysses magnetometer (Balogh et al., 1992). By substituting into the 1.h.s. of Eq. (1) the sixteen measured values of the differential flux, a set of sixteen equations involving the unknown coefficients $J_{0}, \mathbf{A}_{l}$ and $A_{2}$ is obtained. As the number of equations available is larger than the number of unknowns, we can use a least square fit procedure to obtain the required coefficients.

We call $J_{0}$ the omnidirectional flux, $\mathbf{A}_{l}$ the first order anisotropy vector and $A_{2}$ the second order anisotropy. We examine the anisotropies in the solar wind frame. To transform to the solar wind frame of reference we subtract the solar wind bulk flow anisotropy (ComptonGetting anisotropy), calculated from the equation (Ipavich, 1974):

$\mathbf{A}_{l}=\frac{2(\gamma+1)}{v_{i}} \mathbf{V}_{s w}$

where $\mathbf{V}_{s w}$ is the solar wind velocity, measured by the SWOOPS instrument (Bame et al., 1992), $\gamma$ is the spectral index of the ATs ion flux energy spectrum (assuming the latter can be fitted by a curve $E^{-\gamma}$ ) and $v_{i}$ is the ion speed. We then resolve the anisotropy into magnetic field aligned and transverse components.

\section{Results}

The results of the anisotropy analysis for days 327-347 of 1998 are presented in Fig. 1, for the sectored channel of proton energy in the range $1.3-2.2 \mathrm{MeV}$. The first four panels show hourly averages of the omnidirectional flux $J_{0}$, the components of $\mathbf{A}_{l}$ parallel and perpendicular to the magnetic field and $A_{2}$. The subsequent three panels give the magnitude, azimuth and meridional angle of the magnetic field in the RTN system, as measured by the Ulysses magnetometer (Balogh et al., 1992), averaged over $512 \mathrm{~s}$. The bottom panel shows 8-min averages of the solar wind speed as measured by the SWOOPS instrument (Bame et al., 1992).

The magnetic field and plasma data show the presence of a very strong forward shock at 01:46 UT on day 331 , with the field magnitude rising from 0.6 to $3.6 \mathrm{nT}$. Another weaker forward shock has been identified at 23:53 UT of day 339. These shocks are indicated on the plot by dotted lines.

A striking feature of this event is the fact that fluxes of energetic protons remain at constant very high levels for approximately 9 days, as can be seen from the $J_{0}$ plot representing the omnidirectional flux.

A second very unusual feature is that, starting around midday on day $335, A_{1 \|}$ and $A_{2}$ take very 


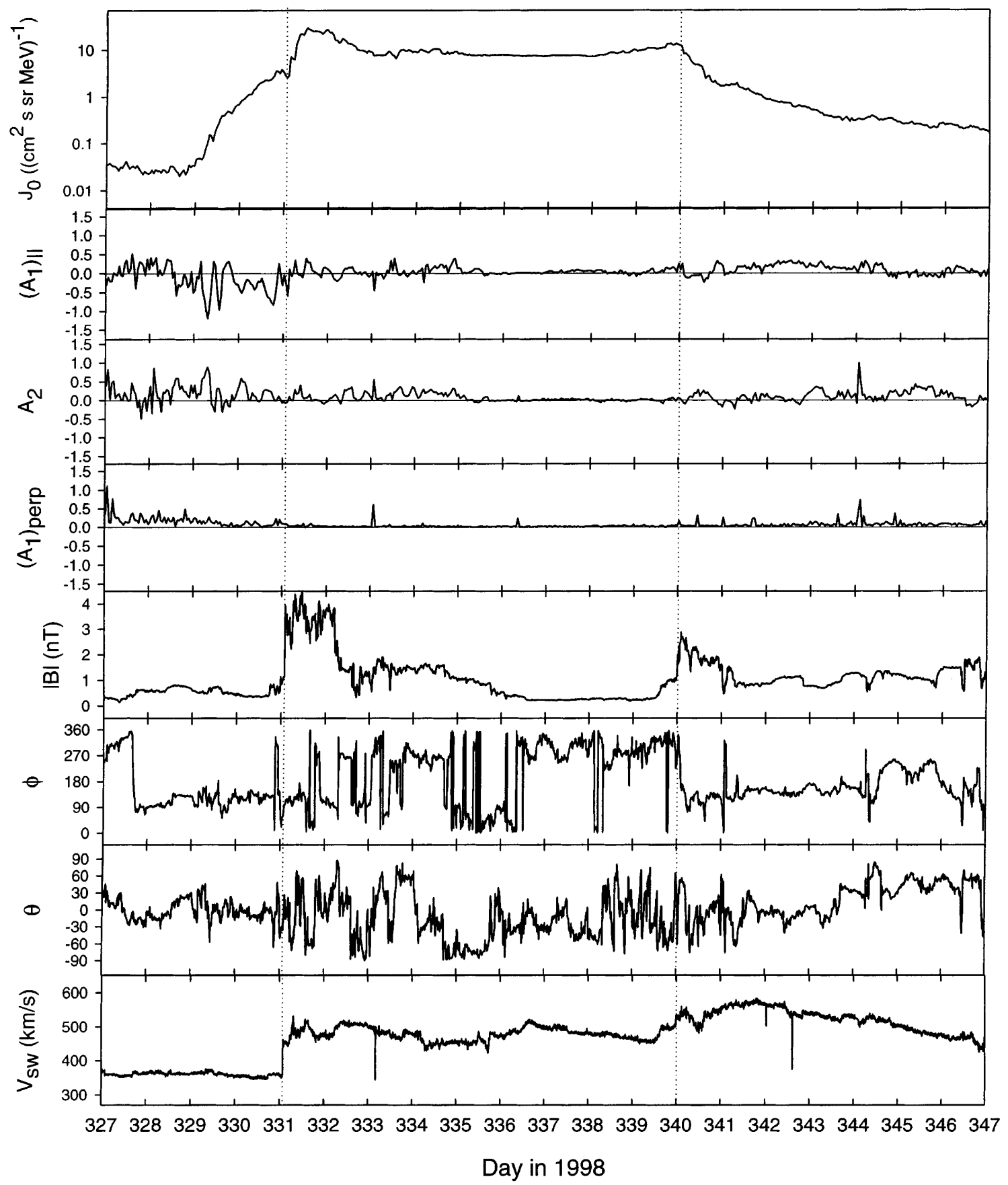

Fig. 1. Overview of the November 1998 event. The omnidirectional flux $J_{0}$, component of the first order anisotropy along the magnetic field, second order anisotropy $A_{2}$ and $\left(A_{1}\right)_{\text {perp }}$ are given. Also the

constant, close to zero, values for a period of almost 4 days. The component of the first order anisotropy perpendicular to the field is almost zero (less than 0.05) during the whole high-flux period. During the zero anisotropy phase the magnetic field magnitude recorded is about $0.3 \mathrm{nT}$ with little variability. During the time interval considered, planar magnetic structures are observed, i.e. periods during which the field direction remains within a plane (Jones, personal communication 1999). In this case the plane contains the nominal Parker spiral direction. magnetic field magnitude, azimuth $\theta$ and meridional angle $\phi$ in the RTN system and finally the solar wind speed are shown

The interpretation of this event is not straightforward. The onset phase of the event, lasting through days $328-330$ is detailed in Fig. 2, where $2048 \mathrm{~s}$ averages of telescope 1 spin-averaged fluxes are presented in the first panel (for protons) and last one (for $\alpha$-particles). The second and third panel give the solar wind proton and $\alpha$-particle density as detected by the SWOOPS instrument. High-energy protons seem to reach the spacecraft earlier than low-energy ones, although it is difficult from this plot to deduce the time delay quantitatively. We also notice from Fig. 2 


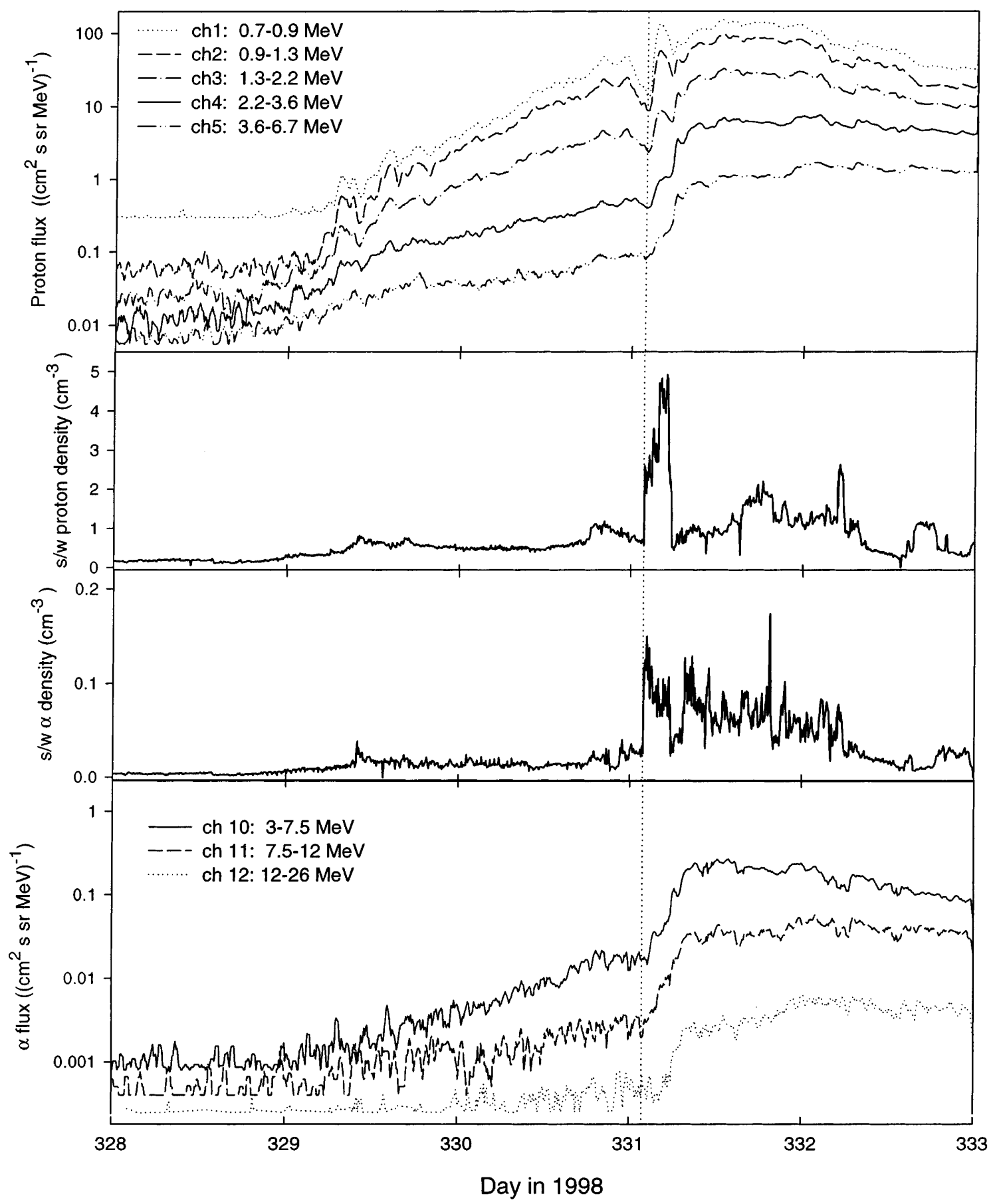

Fig. 2. Onset phase of the November 1998 event. In the first panel ATs spin-averaged proton fluxes for five energy channels are given. The second and third panel show the solar wind proton and $\alpha$-particle

density from the SWOOPS instrument. In the lowest panel are the ATs spin-averaged $\alpha$-particle fluxes for three energy channels

that just after the shock passage on day 331 proton counts in the low-energy channels exihibit a correlation with the solar wind proton density, while at energies higher than $2.2 \mathrm{MeV}$ this is not the case. Proton fluxes show a decrease at the shock location, unlike what happens for typical shock acceleration events, in which fluxes peak at the shock. Also we notice that the $\alpha$-particle time profiles are qualitatively different from the proton ones in that fluxes upstream are much lower than downstream.

From the second and sixth panels of Fig. 1, the anisotropy during the onset phase is seen to be antiSunward. In fact, during days 328-330 the magnetic field azimuth $\phi$ remains close to $90^{\circ}$, corresponding to the field pointing towards the sun, so that a negative value of $A_{1 \|}$ indicates anti-Sunward flow. We observe at this point that at times where $A_{1 \|}$ is nonzero and is in magnitude greater than or equal to $A_{2}$, particle motion is mainly one-directional, parallel or anti-parallel to the field, assuming negligible perpendicular anisotropy. 
The analysis of the anisotropy results close to the shock on day 331 is complicated by the fact that the field azimuth $\phi$ flips to the reverse polarity for a few hours late on day 330. We can say however that, from the sign of $A_{1 \|}$, there is a one-directional flow in the antiSunward direction upstream of the shock, and a smaller on average Sunward flow downstream of the shock, prior to the zero anisotropy period.

The zero anisotropy phase sets in at 16:30 UT on day 335 , at a time when the magnetic field magnitude is $0.86 \mathrm{nT}$ and is decreasing. The magnitudes of $A_{1 \|}$ and $A_{2}$ remain smaller than 0.03 until the end of day 336 , when a small increase to values around 0.05 takes place. The field magnitude reaches a minimum at this time, being of $0.22 \mathrm{nT}$. Anisotropy magnitudes remain smaller than 0.08 for the whole of days 337 and 338, until approximately midday on day 339 , when they increase to values around 0.15 prior to the passage of the second shock. At this point the direction of particle flow is anti-Sunward.

Measurements of the zero-anisotropy phase for three energy channels are detailed in Fig. 3. Here omnidirectional fluxes and anisotropies for channels 7 (the same as in Fig. 1), 8 and 9 are given. We observe very similar anisotropy traces in the three channels considered, i.e.

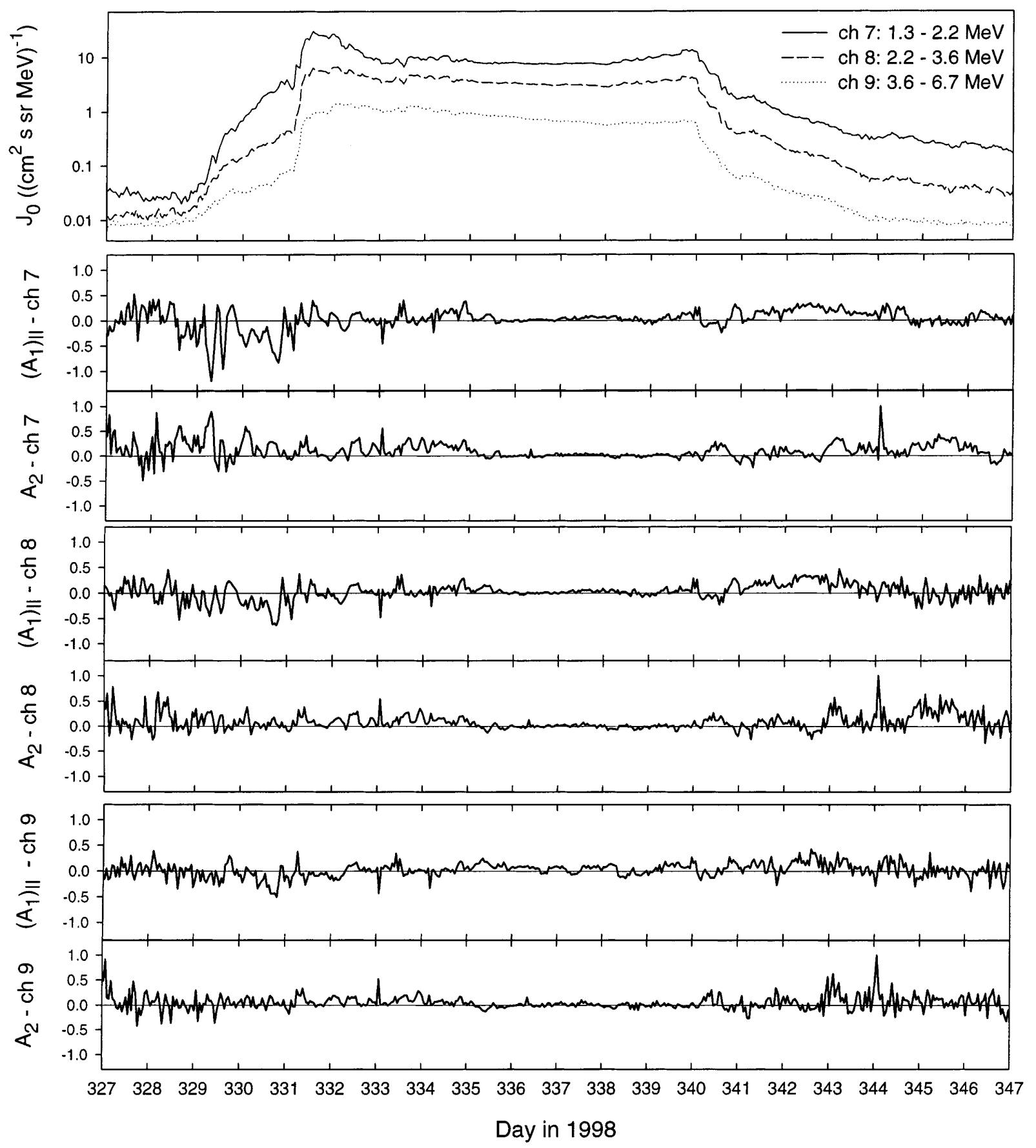

Fig. 3. ATs anisotropy results for three sectored energy channels 
protons in the energy range $1.3-6.7 \mathrm{MeV}$ behave as a well-defined population. In particular, the zero anisotropy phase is clearly visible in all three channels. In the $A_{1 \|}$ trace for the highest energy channel, however, larger anisotropies are observed. There is a possibility that this might be a noisier signal related to low counts.

\subsection{Error on anisotropy coefficients}

An important issue in the discussion of the zero anisotropy phase is that of the error on the anisotropy coefficients as determined from the ATs measurement. This is determined by the error on the counts detected by the two telescopes during the averaging period and by the goodness of the spherical harmonic fit used to find the anisotropies.

The results of this study were obtained by adding ATs counts over a period of $1 \mathrm{~h}$. Typical counts for each sector of channel 7 for this accumulation time are of 2400 for T1 and 2000 for T2. The error, given by $N^{1 / 2}$, where $N$ is the number of counts, is therefore of about $2 \%$ for $\mathrm{T} 1$ and $2.3 \%$ for $\mathrm{T} 2$.

If these errors on the counts are input to the spherical harmonic analysis routine, an error on the coefficients of the fit can be obtained by calculation of the diagonal elements of the covariant matrix. For day 336, e.g., we find that the error on the largest component of the first order anisotropy, the one along the radial direction from the Sun, is typically of $4.6 \%$. At this point we are talking about errors on the anisotropy as measured in the spacecraft frame. The subtraction of the ComptonGetting anisotropy as given by Eq. (2) does not increase the error on the measurement as the solar wind speed, ion velocity and spectral index are determined to a higher accuracy than the calculated anisotropy coefficients.

We conclude, therefore, that the error on the first order anisotropies derived from channel 7 counts is typically $5 \%$ or less.

\subsection{Speed of scattering centres}

The results of the anisotropy analysis can be looked at also from a slightly different perspective, following the approach of Tan et al. (1988). These authors analyse particle data from the ISEE 3 spacecraft for several interplanetary shocks and assume that there exists a reference frame in which the particle distribution function is isotropic and its energy dependence can be approximated by a power law. They argue that this frame is the one co-moving with the waves producing particle pitch-angle scattering and denote its velocity with respect to the solar wind frame as $\mathbf{V}_{s c}$. They find that this vector is Sunward directed and has in some, but not all, cases a magnitude close to the Alfven speed.

To compare our measurement with the results of Tan et al. (1988) we first derive $V_{s c}^{\|}$, the projection along the magnetic field direction of the scattering centres velocity, from our $A_{1 \|}$ using the Compton-Getting expression:
$V_{s c}^{\|}=\frac{v_{i}}{2(\gamma+1)} A_{1 \|}$

To a first approximation we can neglect the perpendicular anisotropy and say that $\left|V_{s c}^{\|}\right|$is the magnitude of $\mathbf{V}_{s c}$. A positive value of $V_{s c}^{\|}$means that the scattering centres are moving along the magnetic field direction, while a negative one means they are moving antiparallel to the field. It is actually preferable to describe this motion in terms of Sunward or anti-Sunward; we therefore look at the magnetic field direction $\phi$ and multiply $V_{s c}^{\|}$by -1 for the Sunward field and by 1 for anti-Sunward field. Positive values of the quantity $V_{s c}^{*}$ thus obtained correspond to anti Sunward motion of the scattering centres and negative values to Sunward motion. A logarithmic plot of $\left|V_{s c}^{*}\right|$ during days 335-341 is given in the last panel of Fig. 4, where solid circles indicate positive values of $V_{s c}^{*}$ and open triangles negative values. For most times during days 335-341 $V_{s c}^{*}$ is positive, indicating anti-Sunward motion. The first three panels of Fig. 4 give the omnidirectional flux and first order field aligned anisotropy for channel 7, and the spectral index $\gamma$. The solid line in the $V_{s c}^{*}$ plot gives the Alfven speed calculated from the equation:

$v_{A}=\frac{B}{4 \pi n_{p} m_{p}}$

where $m_{p}$ and $n_{p}$ are the proton mass and density and the contribution from the solar wind helium has been neglected.

We observe from the last panel of Fig. 4 that at the end of day 335 and for the whole of day 336, when the anisotropy is closest to zero, the speed of scattering centres is very close to the Alfven speed. Starting on day 337, however, a scattering centre velocity of about $200 \mathrm{~km} / \mathrm{s}$ is obtained, much larger than the Alfven speed $v_{A} \sim 30 \mathrm{~km} / \mathrm{s}$. For most of the zero anisotropy phase scattering centres are moving in the anti-Sunward direction, contrary to the result of Tan et al. (1988).

\section{Summary and conclusions}

Anisotropy Telescopes measurements of proton flow directions during the energetic particle event of November 1998 at Ulysses have been reported. This event is unique in that a zero anisotropy phase has been identified within it for all three sectored ATs proton channels. Another unusual feature is the constant high proton flux for a period of nine days in between the passage of two interplanetary forward shocks. During the zero anisotropy phase the magnetic field magnitude is also nearly constant at values of about $0.3 \mathrm{nT}$.

The interpretation of the measurement here reported is not straightforward. A possible scenario for the November 1998 event put forward by Lario et al. (J. Geophys. Res., submitted 2000) is as follows. Association of the two forward shocks with two observed CMEs yields information that they have similar transit speeds (around $700 \mathrm{~km} / \mathrm{s}$ ). It is possible therefore that energetic particles might have remained confined within the region between the two shocks, bouncing back and 


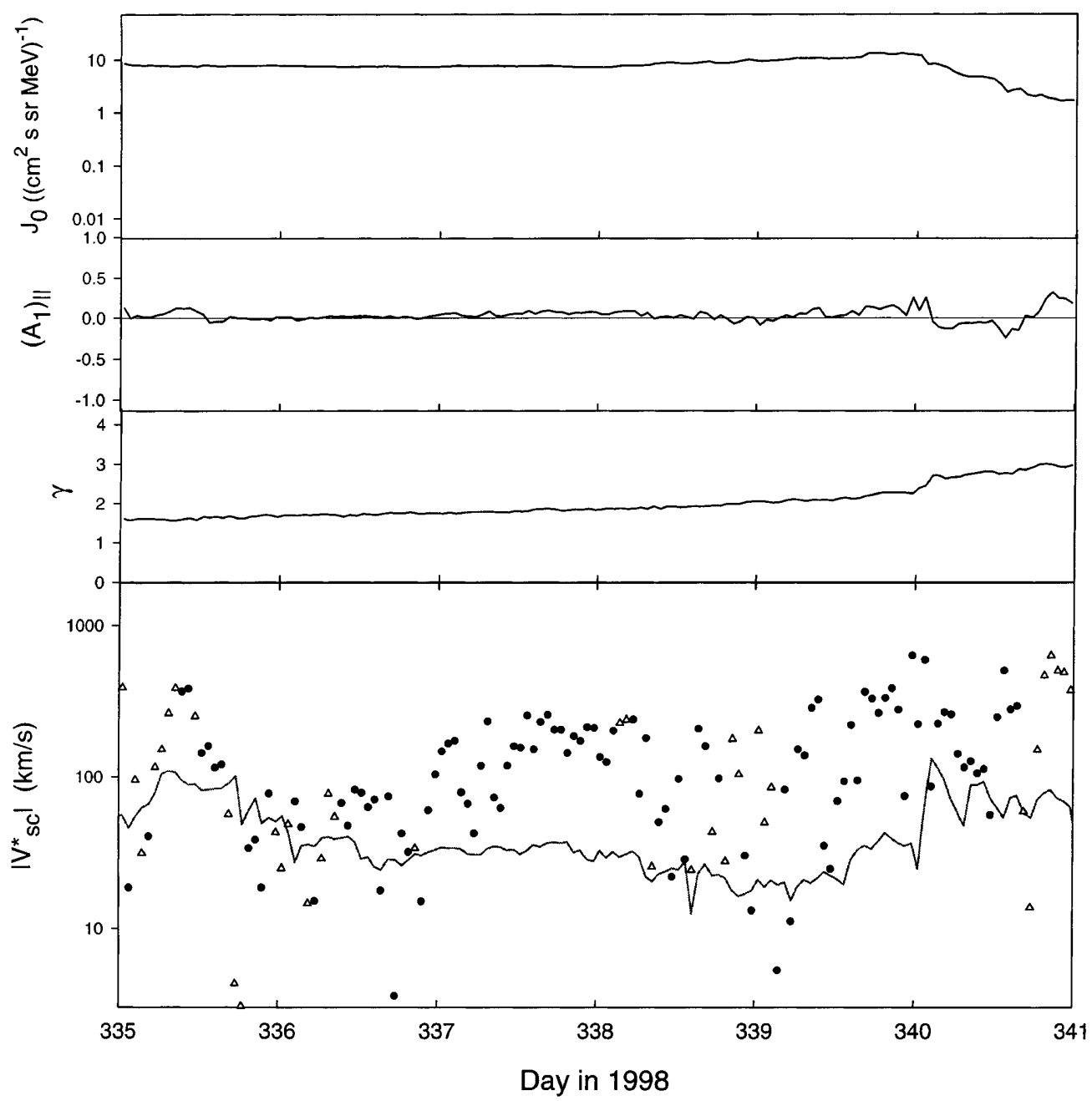

Fig. 4. Detail of the zero anisotropy phase and calculation of the speed of the frame of scattering centres. For channel 7 are shown the omnidirectional flux $\mathbf{J}_{0},\left(A_{l}\right)_{\|}$, the spectral index $\gamma$ and the speed of scattering centres $\left|V_{s c}^{*}\right|$. In the lowest plot solid circles indicate anti-Sunward motion of the scattering centres and open triangles Sunward motion forward between them. It seems likely that these particles were accelerated during a series of solar events taking place during days 326-328, including several Xclass flares and a halo CME at 2:30 UT on day 328. The anisotropy during the onset phase is consistent with this hypothesis.

The reason for the zero anisotropy phase detected by the ATs on days 335-339 remains unclear. If, as proposed by Lario et al. (J. Geophys. Res., submitted 2000), particles were trapped in the region between the two forward shocks, wave-induced pitch-angle scattering might have been particularly effective in isotropizing the particles. If waves were responsible for the pitch-angle scattering which produces the isotropic proton distribution function, according to our measurement these would be moving in the antiSunward direction in the solar wind frame. Their speed in this frame could at times be of the order of the Alfven speed, and at other times up to six times greater.

Finally, the zero anisotropy phase within the event is an indication of good intercalibration of the two ATs telescopes. The latter point is discussed in more detail in the following Appendix.

Acknowledgement. Topical Editor E. Antonucci thanks R. Marsden for his help in evaluating this paper.

\section{Appendix: telescopes intercalibration}

We wish to point out the implications of the zero anisotropy results on the issue of intercalibration between the two telescopes of which the ATs system consists.

We observe first of all that our result that the anisotropy is zero in the solar wind frame means that the anisotropy measured by the system in the spacecraft frame is the Compton-Getting anisotropy. This anisotropy results from the particle population in the solar wind being convected through the spacecraft frame of reference, and is given by Eq. (2).

The Compton-Getting anisotropy is directed along the radial direction in a Sun-centred system of coordinates. Because the spacecraft spin axis is always directed towards the Earth, and Ulysses is at 5.2 AU from the Sun, the radial direction and spacecraft axis are almost coincident, to within $11^{\circ}$. Consequently, a radially directed anisotropy such as the Compton-Getting anisotropy produces an approximately constant number of counts in the eight sectors of each telescope. Because of the inclination of the two telescopes with respect to the spin axis, the actual value of counts in the sectors of telescope 1 is going to be higher than in those of telescope 2, the ratio of the two depending on the magnitude of the anisotropy. In fact, assuming that the flux along the direction $\mathbf{e}_{v}$ can be written as:

$$
J\left(E, \mathbf{e}_{v}\right)=J_{0}(E)\left[1+\mathbf{A}(E) \cdot \mathbf{e}_{v}\right]
$$

where $\mathbf{A}$ is a radially directed anisotropy, we can write:

$J(E, \alpha)=J_{0}(E)[1+A(E) \cos \alpha]$ 
where $\alpha$ now indicates the angle between the radial direction and the direction of particle incidence into a detector. Considering that in the ATs system T1 corresponds to $\alpha=60^{\circ}$ and T2 to $\alpha=145^{\circ}$, we find the following expression for the ratio of fluxes detected in $\mathrm{T} 1$ and $\mathrm{T} 2$ :

$\frac{J_{T 1}}{J_{T 2}}=\frac{1+0.5 \mathrm{~A}}{1-0.82 \mathrm{~A}}$.

From this equation the magnitude of the radial anisotropy $A$ can be expressed in terms of the ratio $J_{T 1} / J_{T 2}$.

Now, in a case in which the only proton anisotropy in the spacecraft frame is due to the Compton-Getting effect, an incorrect intercalibration of the two telescopes of the ATs system would give rise to an incorrect estimate for the magnitude of anisotropy in the spacecraft system $A$. When subtracted from the magnitude of the Compton-Getting anisotropy as calculated from Eq. (2), this would lead to constant nonzero anisotropy in the solar wind frame. From these considerations we conclude that the fact that, in our case, there is constant zero anisotropy in the solar wind frame is an indication of correct intercalibration between the two telescopes.

We also wish to point out that the fact that $\left(A_{l}\right)_{\text {perp }}$ is zero during the whole high flux period is itself sufficient to claim good intercalibration. In fact, even in the presence of particle streaming along the magnetic field lines, the component of the anisotropy perpendicular to the field is expected to be zero in the solar wind frame, within the assumption that there are no significant spatial gradients perpendicular to the magnetic field.

\section{References}

Bame, S. J., D. J. McComas, B. L. Barraclough, J. L. Phillips, K. J. Sofaly, J. C. Chavez, B. E. Goldstein, and R. K. Sakurai, The Ulysses solar wind plasma experiment, Astron. Astrophys. Suppl. Ser., 92, 237-265, 1992.

Balogh, A., T. J. Beek, R. J. Forsyth, P. C. Hedgecock, R. J. Marquedant, E. J. Smith, D. J. Southwood, and B. T. Tsurutani, The magnetic field investigation on the Ulysses mission: instrumentation and preliminary scientific results, Astron. Astrophys. Suppl. Ser., 92, 221-236, 1992.
Ipavich F. M., The Compton-Getting effect for low energy particles, Geophys. Res. Lett., 1, 149-152, 1974.

Lario, D., R. G. Marsden, T. R. Sanderson, M. Maksimovic, A. Balogh, R. J. Forsyth, R. P. Lin, and J. T. Gosling, Ulysses and WIND particle observations of the November 1997 solar events, Geophys. Res. Lett., 25, 3469-3472, 1998.

Marsden, R. G., D. Lario, T. R. Sanderson, M. Maksimovic, A. Balogh, S. Dalla, and R. J. Forsyth, On the energetic particle fluxes at $5 \mathrm{AU}$ associated with the April/May 1998 solar activity: Ulysses observations, in Solar Wind Nine, Eds. S.R. Habbal, R. Esser, J.V. Hollweg and P.A. Isenberg, AIP Conf. Proc. 471, 609-612, 1999.

Sanderson, T. R., R. Reinhard, P. van Nes, and K. P. Wenzel, Observations of three-dimensional anisotropies of 35- to 1000$\mathrm{keV}$ protons associated with interplanetary shocks, J. Geophys. Res., 90, 19-27, 1985.

Sanderson, T. R., R. G., Marsden, K. P. Wenzel, A. Balogh, R. J. Forsyth, and B. E. Goldstein, Ulysses high latitude observations of ions accelerated by corotating interaction regions, Geophys. Res. Lett., 21, 1113-1116, 1994.

Simnett, G. M., K. A. Sayle, E. C. Roelof, and S. J. Tappin, Co-rotating particle enhancements out of the ecliptic plane, Space Sci. Rev., 72, 327-330, 1995.

Simpson, J. A., J. D. Anglin, A. Balogh, M. Bercovitch, J. M. Bouman, E. E. Budzinski, J. R. Burrows, R. Carvell, J. J. Connell, R. Ducros, P. Ferrando, J. Firth, M. Garcia-Munoz, J. Henrion, R. J. Hynds, B. Iwers, R. Jaquet, H. Kunow, G. Lenz, R. G. Marsden, R. B. McKibben, R. Muller-Mellin, D. E. Page, M. Perkins, A. Raviart, T. R. Sanderson, H. Sierks, L. Treguer, A. J. Tuzzolino, K. -P. Wenzel, and G. Wibberenz, The Ulysses cosmic ray and solar particle investigation, Astron. Astrophys. Suppl. Ser., 92, 365-399, 1992.

Staines, K., A. Balogh, S. W. H. Cowley, T. M. Edwards, R. J. Forsyth, and R. J. Hynds, Ulysses observations of noncorotational flows in the outer dayside Jovian magnetosphere, Planet. Space Sci., 41, 931-946, 1993.

Tan L. C., G. M. Mason, G. Gloecker, and F. M. Ipavich, Downstream energetic protons and alpha particles during quasi-parallel interplanetary shock events, J. Geophys. Res., 93, 7225-7243, 1988. 